\title{
Experimental Extraction of Effective Refractive Index and Thermo-Optic Coefficients of Silicon-On-Insulator Waveguides using Interferometers
}

\author{
Sarvagya Dwivedi, Alfonso Ruocco, Michael Vanslembrouck, Thijs Spuesens, Peter Bienstman, Pieter Dumon, \\ Thomas Van Vaerenbergh and Wim Bogaerts
}

\begin{abstract}
We propose and demonstrate an accurate method of measuring the effective refractive index and thermo-optic coefficient of Silicon-On-Insulator waveguides in the entire $\mathrm{C}$ band using three Mach-Zehnder Interferometers. The method allows for accurate extraction of the wavelength dispersion and takes into account fabrication variability. Wafer scale measurements are performed and effective refractive index variations are presented for three different waveguide widths: 450,600 and 800 $\mathrm{nm}$, for the TE polarization. The presented method is generic and can be applied to other waveguide geometries and material systems and for different wavelengths and polarizations.
\end{abstract}

Keywords-Waveguides, effective refractive index, Wavelength filtering devices, thermo-optic coefficients.

\section{INTRODUCTION}

Silicon photonics is a rapidly growing field with applications from optical interconnects to bio-sensing. The silicon-oninsulator (SOI) platform has been a promising platform for mass scale production of silicon photonics devices since it can use complementary metal-oxide-semiconductor (CMOS) process technology for fabrication. This offers a scaling avenue to large-volume production at an affordable cost.

SOI can be used to define waveguides with a high refractive index contrast between core and cladding. This enables dense integrated photonic circuits with submicron waveguide cores and sharp bends. However, the high index contrast and small waveguide dimensions can translate small variations in waveguide width and thickness into a large fluctuations in the effective refractive index [1]. This is a potential weakness to this platform and extremely precise fabrication is required to achieve a good yield. Also, the high refractive index contrast makes the waveguide properties quite dispersive with wavelength.

\footnotetext{
Sarvagya Dwivedi, Alfonso Ruocco, Michael Vanslembrouck, Thij Spuesens, Peter Bienstman, Pieter Dumon, Thomas Van Vaerenbergh and Wim Bogaerts are with Photonics Research Group, Department of Information Technology Ghent University - imec and Center for Nano- and Biophotonics, Sint-Pietersnieuwstraat 41, B-9000 Ghent, Belgium. e-mail: sarvagya.dwivedi@intec.ugent.be

Pieter Dumon and Wim Bogaerts are also with:Luceda Photonics, Noordlaan 21, 9200 Dendermonde, Belgium

T. Van Vaerenbergh is now with: Hewlett-Packard Laboratories, 1501 Page Mill Road, Palo Alto, CA 94304, USA.

Manuscript received June 01, 2015; revised
}

In order to design effectively in such a waveguide platform, the exact value of the optical properties should be known, including their wavelength dependence and the statistics of their variability. Many optical properties, such as waveguide loss, can be collected from optical measurements on fabricated devices. But this is difficult for the effective refractive index $\left(n_{e f f}\right)$ of the waveguide.

The effective refractive index is a very critical parameter in the design of wavelength filters [2], [3]. It determines the exact condition of constructive interference, and therefore the central operating wavelength of a filter. Effective index changes can therefore cause a wavelength shift of a filter: in silicon wire waveguides this shift is approximately $1 \mathrm{~nm}$ for $1 \mathrm{~nm}$ of waveguide width change and around $1.4 \mathrm{~nm}$ for $1 \mathrm{~nm}$ change in waveguide thickness. Similar to geometry variations, local and environmental temperature fluctuations can cause a change in effective index: since silicon has a high thermo-optic (TO) coefficient of $1.86 \times 10^{-4} \mathrm{~K}^{-1}$, this can result in a filter wavelength shift of $80 \mathrm{pm} / \mathrm{K}$ [4].

Given the extreme sensitivity of a silicon wire waveguide to the exact geometry, it is important to accurately characterize the effective index, including its dispersion, without perturbing the actual waveguide geometry. Existing methods to extract the dispersion such as prism coupling are either not suited for high refractive index waveguides, or actively perturb the waveguide [5]. Non-invasive methods such as ellipsometry cannot be easily applied to narrow waveguides. Techniques using scattering with weak higher-order periodic perturbations effectively perturb the waveguide, but also change the local pattern density, and thus could even influence the fabrication process [1].

Methods which rely on relative features in the transmission spectrum of interferometric devices will measure the group index $n_{g}$ rather than the effective index $n_{\text {eff }}$ [6]. Because silicon wire waveguides are very dispersive, $n_{g}$ and $n_{e f f}$ are very different, and highly wavelength dependent. Knowing the group index, it is theoretically possible to integrate the effective index, but this can result in an ambiguous solution. Therefore, a method is needed to extract the effective index (as well as the group index) unambiguously and including their wavelength dispersion and temperature dependence.

Simulation of a waveguide cross section to extract the 
waveguide mode properties is also possible, but at best it gives a good estimate of the effective index. In practice, the geometry of a buried silicon wire is hard to measure experimentally, even with high-resolution SEM. Also, boundary effects in the material properties can also affect the effective index but cannot be perceived by visual or SEM inspections. However, simulations can provide a first estimate which we also use in our method as a starting point to improve the accuracy.

We present a simple approach to accurately measure the $n_{\text {eff }}$ of a silicon photonics wire. Our method uses three Mach-Zehnder interferometers (MZI) designed with the same photonic wire located as close as possible to each other. MZIs are good candidates as they are simple in design, linear in phase response and flexible in choosing the filter order. Other methods of parameter extraction using ring resonators can suffer from nonlinearity in terms of phase and higher input optical power due to their resonant nature [7], [8]. Also, the roundtrip length of a ring resonator is a combination of couplers, bends and straight waveguides, and the extracted $n_{\text {eff }}$ and $n_{g}$ will be averaged over these different sections [9].

In our method we use two MZIs with a low order $m$, i.e. a large free spectral range (FSR), which allow us to estimate the order of the filter correctly within the C-band. The third MZI has a much higher order and a small FSR, which helps us to extract the wavelength dependence of the group index $\left(n_{g}\right)$. To extract the TO coefficients, we measure the same MZIs at different temperatures and then extract the wavelength sensitivity of the spectrum with respect to temperature. We also perform and present wafer-scale measurements for three different waveguide widths of $450 \mathrm{~nm}, 600 \mathrm{~nm}$ and $800 \mathrm{~nm}$ respectively. Our method is generic in nature and should work for all integrated waveguide platforms. We already reported preliminary results of this method [10]. In this paper the method is fully elaborated and demonstrated for different waveguide sections, including wafer-scale statistics and thermo-optic coefficients extraction.

\section{METHOD}

The effective refractive index of an SOI waveguide at a given wavelength is a function of the materials, the waveguide cross-sectional geometry and environmental temperature. We can conceptually simplify this as

\section{A. Effective Index Model}

$$
n_{\text {eff }}(\lambda)=f(w, h, T)
$$

where $w, h$ is waveguide width and thickness respectively and $T$ is the environment temperature.

The eigenmode simulations for the initial estimate of the SOI waveguide mode are performed by the finite element method (FEM) from COMSOL, taking a wavelength and temperature dependent material model. The wavelength and temperature dependent refractive index material model of silicon and oxide at room temperature is given by the Sellmeier equation [11], [12], [13] :

$$
n_{\lambda, T}^{2}-1=\sum_{i=1}^{3} \frac{S_{i}(T) \lambda^{2}}{\lambda^{2}-\lambda_{i}^{2}(T)}
$$

where $S_{i}(T)$ is a temperature dependent Sellmeier coefficients. At $\lambda=1550 \mathrm{~nm}$ and room temperature, the value of $n_{S i}$ is 3.4777 and for $n_{\mathrm{SiO} 2}$ is 1.4440 . The thermo-optic coefficient $\left(\frac{d n}{d T}\right)$ at $1550 \mathrm{~nm}$ is $1.86 \times 10^{-4} K^{-1}$ for silicon and $1.0 \times 10^{-5} K^{-1}$ for oxide, here $T$ is the temperature in Kelvin $(K)$.

\section{B. Extracting the Effective Index from a single MZI}

In any given wavelength filter, as in our MZI, we get constructive interference at the output when

$$
m \cdot \lambda_{\text {res }}=n_{\text {eff }}\left(\lambda_{\text {res }}\right) \cdot \Delta L
$$

where, $\lambda_{\text {res }}$ is resonance wavelength, $m$ is the filter order, $n_{\text {eff }}\left(\lambda_{\text {res }}\right)$ is effective index of the waveguide at that wavelength and $\Delta L$ is the physical path length difference.

From Eq. (3), if we know the resonance wavelength and the filter order, we can extract the effective index at that wavelength. In normal practice, we designed the filter with a known order $m$, using a prior estimate of $n_{e f f}$. However, if the possible deviations in $n_{\text {eff }}$ from our design estimate become too large, we cannot be sure about the order. Identifying the order of a filter response is essential, as this gives us an absolute reference for the calculation of the effective index. For a given design order $m$, the largest change $\Delta n_{\text {eff }}$ that we can allow to successfully identify the same order $m$ of the fabricated design is

$$
\Delta n_{e f f}=\frac{2}{2 m+1}
$$

We can assume that in first order, $\Delta n_{e f f}$ is due to a change in width $\Delta w$ and height $\Delta h$ :

$$
\Delta n_{e f f}=\frac{d n_{e f f}}{d w} \cdot \Delta w+\frac{d n_{e f f}}{d h} \cdot \Delta h
$$

Here, $d n_{e f f} / d w$ and $d n_{e f f} / d h$ is the change in $n_{e f f}$ due to waveguide width and thickness respectively at a given temperature. These trends we can quite reliably extract from simulations. The maximum allowed $\Delta n_{e f f}$ for a wavelength filter of a given order is shown in Fig. 1. We see that for higherorder filters, it becomes difficult to estimate the interference order unless the fabrication process is very precise.

Fig. 1 also shows the maximum $\Delta n_{\text {eff }}$ for three different waveguides widths i.e. $450 \mathrm{~nm}, 600 \mathrm{~nm}$ and $800 \mathrm{~nm}$ for a fabrication process with an accuracy of $\pm 20 \mathrm{~nm}$ for waveguide linewidth and $\pm 5 \mathrm{~nm}$ for waveguide thickness which is estimated from Eq. (5) [1]. As can be expected, the wider waveguides will suffer less from small absolute linewidth variations than the narrow waveguide. For the $450 \mathrm{~nm}$ wide waveguide the field strength of the fundamental TE mode is much stronger on the side wall boundaries than width the 600 $\mathrm{nm}$ and $800 \mathrm{~nm}$ waveguides. the specified variation of $\pm 5 \mathrm{~nm}$ on the waveguide thickness does not affect the TE mode as 


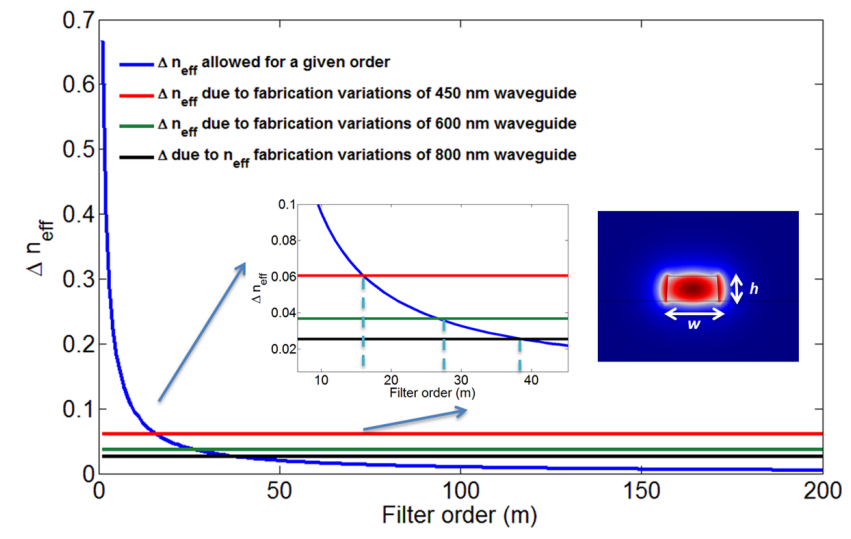

Fig. 1: The estimated $\Delta n_{e f f}$ allowed and maximum fabrication variations for three different waveguides 450,600 and $800 \mathrm{~nm}$ for increasing filter order $m$. Inset 1: Zoomed plot showing the region of operation of which order to choose when there maximum fabrication in a different waveguides. Inset 2: Simulated 2D TE mode profile of $450 \mathrm{~nm}$ wide and $215 \mathrm{~nm}$ SOI waveguide with oxide as top cladding at room temperature.

much, but it will have a much stronger impact on the TM mode. For this work we focus on the fundamental TE mode but the method can be extended to the TM mode.

The intersection of the 3 horizontal traces with the blue trace gives us the maximum order of a wavelength filter where we can still identify the order $m$ with confidence. For 450, 600 and $800 \mathrm{~nm}$, the values of $m$ are 17, 27 and 38 respectively. When designing a filter to use for refractive index extraction, we should pick an order below these values.

When we design our filter with a sufficiently low order, the possible changes in the refractive index will be low enough such that

$$
\begin{aligned}
& n_{e f f}^{(m-1)}+\Delta n_{e f f}<n_{e f f}^{(m)}-\Delta n_{e f f} \\
& n_{e f f}^{(m)}+\Delta n_{e f f}<n_{e f f}^{(m+1)}-\Delta n_{e f f}
\end{aligned}
$$

This means that we can unambiguously associate each $\lambda_{\text {res }}$ of constructive interference with an order $m$. This is shown in Fig. 2, where we simulated an MZI with $450 \mathrm{~nm}$ wide waveguide case for $m=15$ (middle curve). The error bars indicate our initial uncertainty on the $n_{e f f}$. The top and bottom curve then show the extracted index if we would have made an error of \pm 1 in the order $m$. It is clear that the curves do not overlap, even when taking into account the uncertainty. This means that we can confidently use a filter with this order for the waveguides in this fabrication process.

\section{Using high-order MZI}

A low-order MZI allows us to accurately identify the order of interference at $\lambda_{\text {res }}$ and thus the effective index at that wavelength. However, in the typical wavelength range of the C-band around $1550 \mathrm{~nm}$, this will result in at most two peaks and valleys in the transmission spectrum. This makes it difficult to extract an accurate model for the wavelength dispersion.

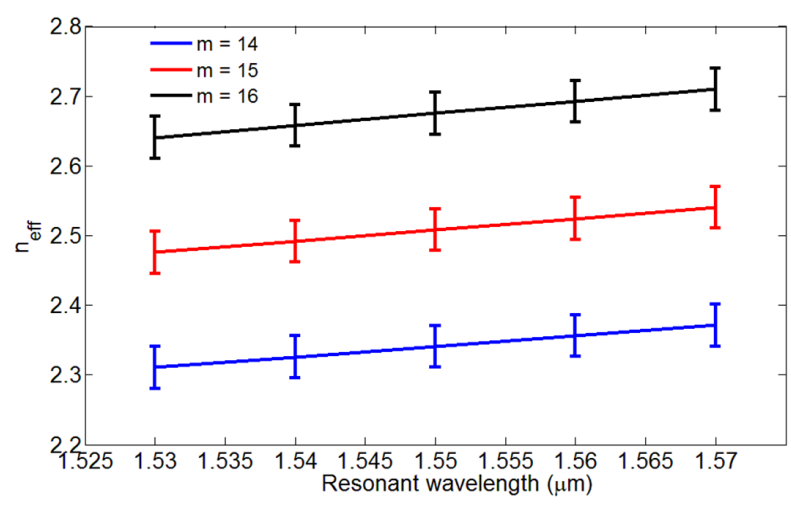

Fig. 2: Simulated effective index for neighboring order at resonant wavelengths for $450 \mathrm{~nm}$ waveguide. Shown error margin is due to the maximum fabrication variations

To compensate for this, we introduce another MZI with a much higher interference order $M$. From this we can get a large number of peaks and valleys in the wavelength band. The FSR of the filter is the difference between two consecutive peaks of the filter:

$$
F S R=\lambda^{2} / n_{g} \cdot \Delta L
$$

From this device, extraction of $n_{g}(\lambda)$ of the waveguide is possible by extracting the wavelength dependent FSR from the transmission measurements. With above these information we can extract the coefficients of modified Taylor expansion in $n_{e f f}(\lambda)$ and $n_{g}(\lambda)$, based on the reference of the known $n_{e f f}\left(\lambda_{0}\right)$ and is given by:

$$
\begin{gathered}
n_{e f f}(\lambda)=a+b \Delta \lambda+c(\Delta \lambda)^{2} \\
n_{g}(\lambda)=a-b \lambda_{0}-c\left(\lambda_{0} \cdot \Delta \lambda\right)
\end{gathered}
$$

where $a=n_{\text {eff }}\left(\lambda_{0}\right), b=\frac{d n_{e f f}}{d \lambda}$ and $c=\Delta \lambda \cdot \frac{d^{2} n_{e f f}}{d \lambda^{2}}$. For the TE waveguide, we can ignore the further terms of the Taylor expansion, since they are comparable to the fitting error for the designed TE-polarized waveguide. For TM waveguides, the higher-order terms would be non-negligible. From low order MZI, $a$ can be extracted without an error. The $b$ and $c$ coefficients are extracted by accurately by higher order filter. Combining these two we have all the coefficients and accurate extraction of $n_{e f f}(\lambda)$ and $n_{g}(\lambda)$.

\section{A second low-order MZI}

Because we use two different devices for extracting a single effective index, there is always the possibility that local variability between the two devices introduces errors in our extraction procedure. To reduce this error, we introduce a second low-order MZI, with an order $m^{\prime}>m$, but still lower than the maximum allowed order needed to identify it unambiguously. We design this second MZI to have the same resonance wavelength as the original MZI: 


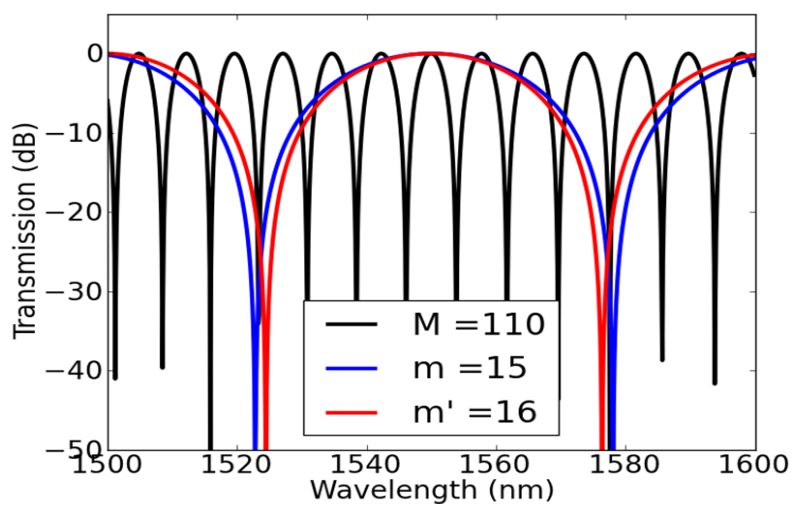

Fig. 3: Simulated circuit transmission of an MZI filter made out of $450 \mathrm{~nm}$ wide and $215 \mathrm{~nm}$ thick SOI waveguide with $m=15, m^{\prime}=16$ and $M=110$ respectively

$$
\frac{\Delta L}{m}=\frac{\Delta L^{\prime}}{m^{\prime}}
$$

We can now estimate the local variations of the effective index $\Delta n_{\text {eff }}$ from the shifts of the two resonances $\lambda_{r e s}$ and $\lambda_{\text {res }}^{\prime}$, which ideally should be zero.

$$
\Delta n_{e f f}=2 \cdot \frac{m^{\prime} \cdot \lambda_{r e s}^{\prime} \cdot \Delta L-m \cdot \lambda_{r e s} \cdot \Delta L^{\prime}}{m^{\prime} \cdot \lambda_{r e s}^{\prime} \cdot \Delta L+m \cdot \lambda_{r e s} \cdot \Delta L^{\prime}}
$$

For our experiments, we chose two MZIs with low order $m=15$ and $m^{\prime}=16$, to satisfy the condition on fabrication variations mentioned in Eq. (4-7) and $M=110$ to get the $n_{g}(\lambda)$ in the wavelength of interest. For this, we used a simulated model of $n_{e f f}$, calculated using a finite-element fully vectorial mode solver, and using a geometry extracted from a cross-sectional SEM image. With this parameters, even with a significant deviation from the model, we are able to still identify the peaks of the right order.

\section{E. Extraction of Thermo-Optic Coefficient}

For extraction of the thermo-optic (TO) coefficient of a waveguide, the wavelength sensitivity with respect to temperature needs to be measured and it is given by [4] :

$$
\left.\frac{d n}{d T}\right|_{\lambda_{r e s}}=\frac{n_{g}}{\lambda_{\text {res }}} \frac{d \lambda}{d T}
$$

The TO coefficient of the waveguide is extracted after first extracting the waveguide group index $n_{g}$ at a given wavelength $\lambda_{m}$ and filter transmission at different temperatures $T$.

An alternative method to extract the TO coefficient is to extract $n_{\text {eff }}\left(\lambda_{\text {res }}\right)$ from the low order MZIs for different temperatures and then use equation (3) directly at a given wavelength.

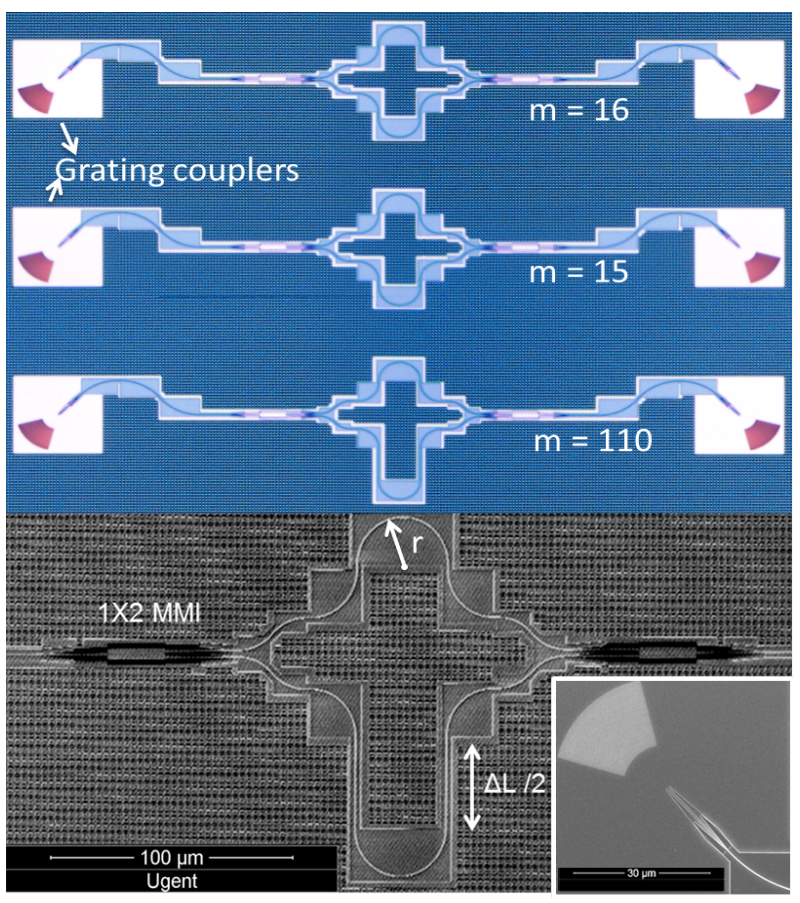

Fig. 4: (a). Camera image of set of fabricated devices for $600 \mathrm{~nm}$ wide waveguide and (b) SEM image of fabricated MZI with $M=110$ showing the MMI, bend radius $r$ and path length difference $\Delta L$. Inset: Low reflection compact grating coupler.

\section{DESIGN AND FABRICATION}

The MZIs are simulated and designed for three different waveguides, i.e. $450 \mathrm{~nm}, 600 \mathrm{~nm}$ and $800 \mathrm{~nm}$ wide respectively and with $215 \mathrm{~nm}$ thick silicon wires for values $m=15, m^{\prime}=$ 16 and $M=110$ respectively. The full eigenmode solutions as discussed in section 2 are fed to the circuit solver CAPHE to get the transmission response at fixed room temperature [14], [15]. The simulated transmission of the three 450nm MZI filters for $m=15, m^{\prime}=16$ and $M=110$ is shown in Fig. 3. As they have been designed for that condition, the three simulations show constructive interference at $\lambda_{\text {res }}$ of $1550 \mathrm{~nm}$.

Devices are designed using the IPKISS by Luceda Photonics design framework, which generates the layouts directly from a parametric description of the MZI [15].

Because the quality of the transmission trace is essential for an accurate extraction, we have designed the test circuits to minimize reflections. There are different contributions of reflection in SOI based wavelength devices. The major contributions to reflection are from the grating couplers to and from the chip. Therefore, we use compact off-axis grating couplers with reduced back reflections [16]. They are designed at an angle of $45 \mathrm{deg}$ to the in-plane fiber axis, and they direct backreflections away from the input waveguide. This reduces back-reflection from $-20 \mathrm{~dB}$ to $-50 \mathrm{~dB}$.

The arms of the MZI are designed with a relatively large bend radius of $20 \mu \mathrm{m}$ to avoid reflections from the straightbend interface and to minimize the loss. The arms are symmetric except for the vertical straight section which incorporates 


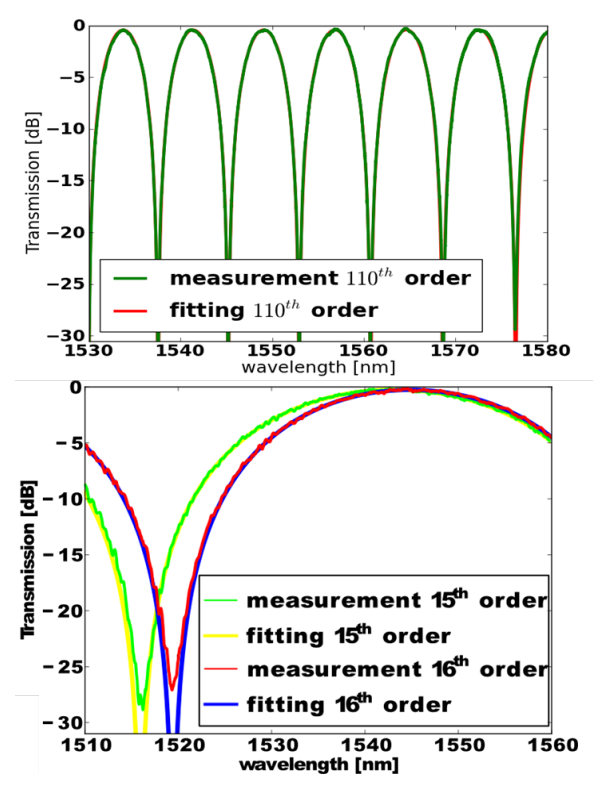

Fig. 5: Measured and fitted normalized spectrum of MZI designed with 450 $\mathrm{nm}$ wide and $215 \mathrm{~nm}$ thick silicon waveguide (a) higher filter order and (b) lower filter order.

the actual delay $\Delta L$. This cancels out possible effects of bendradius dependence of $n_{e f f}$.

Our device focuses on measuring and extracting the $n_{e f f}$ of straight photonic wires. It can be extended to extract the bend mode waveguide refractive index by incorporating the delay line in the bend sections.

The side wall roughness of the waveguide is unavoidable and depends upon the fabrication. This effect is prominent in $450 \mathrm{~nm}$ wide waveguides and gradually decreases for wider waveguides i.e for 600 and $800 \mathrm{~nm}$.

For equal splitting and combining the light, the device uses wide band $1 \times 2$ multimode interference (MMI). By using a symmetric splitter and combiner we reduce the effect of wavelength dependence in directional couplers, and the couplers do not inherently introduce a phase delay between the arms.

The fabrication of our device has been done in the imec 200 $\mathrm{mm}$ CMOS pilot line using silicon-on-insulator wafers with an nominal $220 \mathrm{~nm}$ of silicon and $2 \mu \mathrm{m}$ of buried oxide and 1.25 $\mu \mathrm{m}$ of top oxide [17] . During the processing, approximately $5 \mathrm{~nm}$ of silicon is removed.

Camera images of all three MZIs designed with $600 \mathrm{~nm}$ wide waveguide and SEM image of one of the fabricated MZI with $M=110$ are shown in Fig. 4.

\section{MEASUREMENTS AND AnAlysis}

\section{A. Device Measurements}

The transmission measurements are performed in a thermally controlled environment to eliminate the effect of the high thermo- optic (TO) coefficient of silicon, which can induce a wavelength peak shift about of $80 \mathrm{pm} / \mathrm{K}$. The device

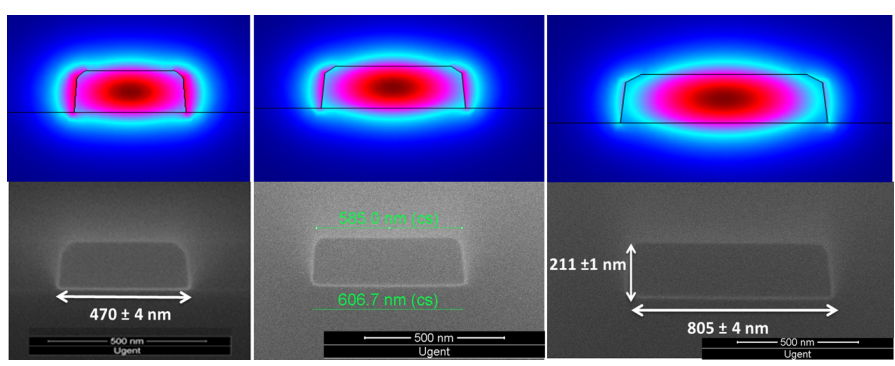

Fig. 6: Simulated mode profile and XSEM image of $470 \mathrm{~nm}, 604$ and $805 \mathrm{~nm}$ wide and 211 thick waveguides.Side wall angles of the trapezoidal waveguides can be easily seen in the SEM

transmission is first normalized to that of a simple straight waveguide to eliminate the grating coupler spectrum. The 10 $\mathrm{dB}$ bandwidth of the grating coupler is around $80 \mathrm{~nm}$ from $1505 \mathrm{~nm}$ to $1585 \mathrm{~nm}$. The on-chip insertion loss of the MZI is less than $0.3 \mathrm{~dB}$.

The normalized transmission spectrum is fitted using nonlinear least square method and cosine square interferometric model given by Eq. $9-10$ as a base function. The fitting procedure minimizes the RMS error between the measured and theoretical transmission trace. For a proper statistical analysis of these errors we use a re-sampling technique. The RMS of the fitting error is used as the standard deviation of a random Gaussian noise model of the measurement perturbation. This procedure is reiterated 1000 times to obtain a statistical relevant data set. The results of these fittings are used to define the standard deviations of the $a, b$ and $c$ coefficients used in eq. (9-refeq:ng) to define $n_{\text {eff }}(\lambda)$ and $n_{g}(\lambda)$.

The measured and fitted the transmission spectra of the 3 MZIs designed for $450 \mathrm{~nm}$ are shown in Fig. 5. However, the measured width and thickness of the waveguide are closer to $470 \pm 4 \mathrm{~nm}$ and $211 \pm 1 \mathrm{~nm}$ respectively, as extracted from SEM inspection. The other two waveguides, designed at 600 $\mathrm{nm}$ and $800 \mathrm{~nm}$, are closer to the designed width, as shown in the SEM images.

The cross-section SEM (XSEM) of the waveguide, the simulated mode profile of all the three waveguides i.e. 470 , 604 and $805 \mathrm{~nm}$ are shown in Fig. 6.

The extracted $n_{e f f}$ and $n_{g}$ for all three waveguides over the entire C-band is shown in Fig. 7.

The extracted $n_{g}$ from the higher order filter and then the $n_{\text {eff }}$ from the low order filter of the different waveguide widths at $1550 \mathrm{~nm}$ is shown in the table I.

TABLE I: Table showing the extracted $n_{e f f}$ and $n_{g}$

\begin{tabular}{|l|l|l|l|l|}
\hline Waveguide & $n_{e f f}$ & $n_{e f f}$ fitting $\sigma$ & $n_{g}$ & $n_{g}$ fitting $\sigma$ \\
\hline $470 \mathrm{~nm}$ & $\begin{array}{l}2.355 \\
\pm 0.002\end{array}$ & $1.8007 \mathrm{e}-6$ & $4.2739 \pm 0.0042$ & $3.963 \mathrm{e}-5$ \\
\hline $602 \mathrm{~nm}$ & $\begin{array}{l}2.534 \\
\pm 0.0035\end{array}$ & $3.806 \mathrm{e}-6$ & $4.0453 \pm 0.0045$ & $4.127 \mathrm{e}-5$ \\
\hline $805 \mathrm{~nm}$ & $\begin{array}{l}2.67 \\
\pm 0.004\end{array}$ & $7.7095 \mathrm{e}-6$ & $3.8902 \pm 0.005$ & $4.321 \mathrm{e}-5$ \\
\hline
\end{tabular}

As shown in the table I the $( \pm)$ contribution in the $n_{\text {eff }}$ and $n_{g}$ extraction comes from the measurement error and the 

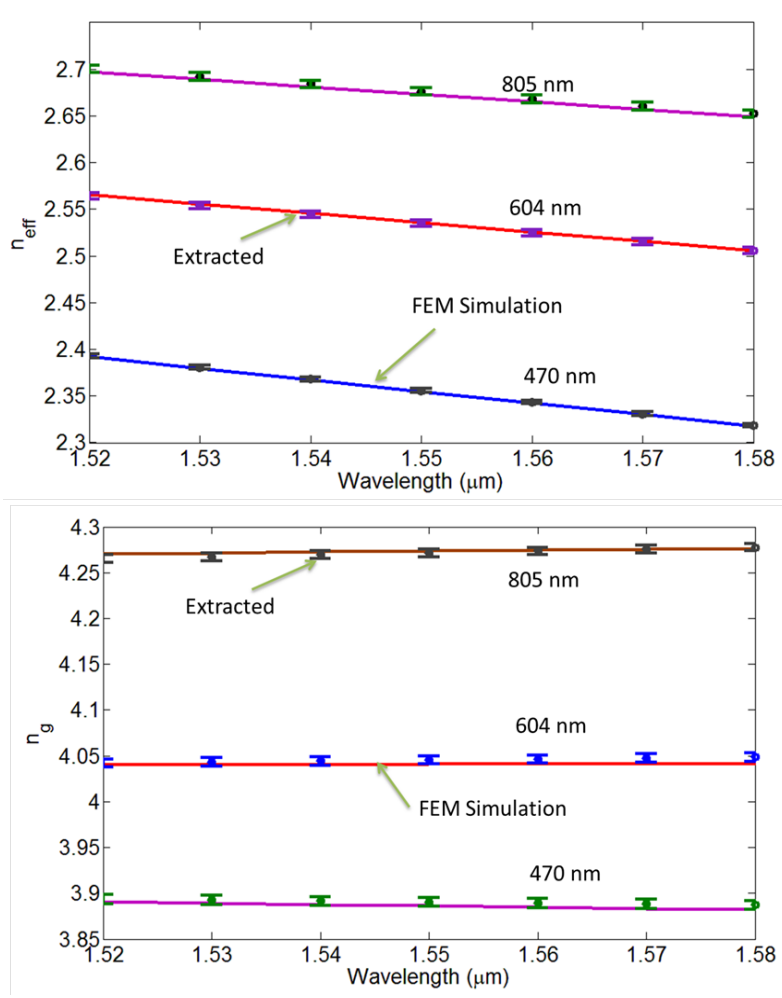

Fig. 7: (a) Simulated and extracted $n_{\text {eff }}$ of 470, 602 and $805 \mathrm{~nm}$ wide waveguides and (b) Simulated and extracted $n_{g}$ over the C-band

standard deviation $(\sigma)$ is from the fitting.

The mismatch between the extracted value and the simulations of the waveguide according to XSEM (with best fitted width, taking into account the uncertainty of the SEM measurement itself) mainly comes from local environmental variations, and fabricated waveguide geometrical non-idealities.

The local variability can be estimated by extracting the $n_{\text {ef } f}$ from $m^{\prime}=16$ as well and compare with $m=15$. The variation of $\Delta n_{e f f}$ for $470 \mathrm{~nm}$ wide wire due to this is around \pm 0.0015 .

\section{B. Thermo-Optic coefficient Measurements}

For the measurements of the TO coefficient $\left(\frac{d n}{d T}\right)$ we placed the fabricated chip on a thermally controlled chuck with a resistive heater and temperature sensor. The MZIs are measured at different a temperature and due this thermal variation underneath the transmission spectrum gets red-shifted. The shifted spectrum is fitted in the similar way as explained in previous section and by Eq. (13) the TO coeff of the waveguides can be extracted. The extracted TO coefficients for all the three waveguides are shown in Fig.8.

The error contribution in TO coefficient extraction can be mainly attributed to small thermal fluctuations in the chuck.

\section{Wafer Scale Measurements}

We characterized these devices over an entire wafer using a fully automatic optical probe station. The wafer map and

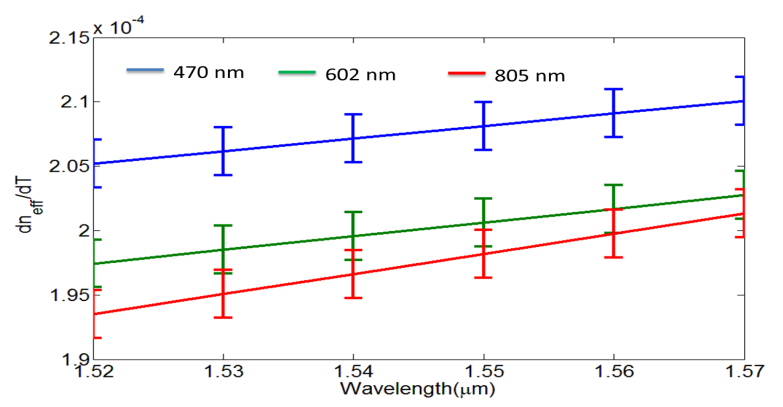

Fig. 8: Extracted $\frac{d n_{e f f}}{d T}$ of 470,602 and $805 \mathrm{~nm}$ wide waveguides
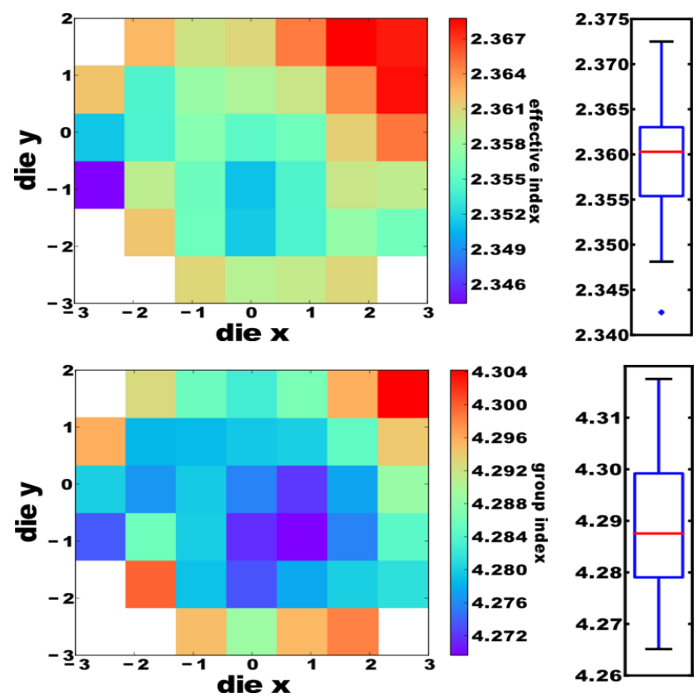

Fig. 9: Wafer map and distribution plot of $470 \mathrm{~nm}$ wide fabricated waveguide at $1550 \mathrm{~nm}$ (a) $n_{\text {eff }}$ and (b) $n_{g}$

distribution plot of extracted $n_{e f f}$ and $n_{g}$ for $470 \mathrm{~nm}, 600 \mathrm{~nm}$ and $800 \mathrm{~nm}$ wide and $211 \mathrm{~nm}$ thick waveguides at $1550 \mathrm{~nm}$ are shown in Figs. 9, 10, and 11 respectively. Across the wafer, for $470 \mathrm{~nm}$ waveguide at $1550 \mathrm{~nm}$, the mean value and standard deviation of $n_{e f f}$ is 2.360 and 0.008 and for $n_{g}$ its 4.287 and 0.018 respectively. For the $600 \mathrm{~nm}$ waveguide the mean value and standard deviation of $n_{e f f}$ is 2.533 and 0.006 and for $n_{g}$ its 4.045 and 0.02 . And, for the $800 \mathrm{~nm}$ wide waveguide, the mean value and standard deviation of $n_{e f f}$ is 2.679 and 0.008 and for $n_{g}$ its 3.924 and 0.01 .

Towards the edge of the wafer map we see the strongest deviations of the extracted effective indices. This can be largely attributed to a deviation in silicon thickness of the SOI wafers near the edge.

\section{Conclusion}

We proposed and demonstrated a generic method for measuring the refractive index of dispersive SOI waveguides. By combining 3 MZIs of low and high order we can extract the wavelength and temperature dependent effective index and the group index unambiguously, and also obtain an estimate for 

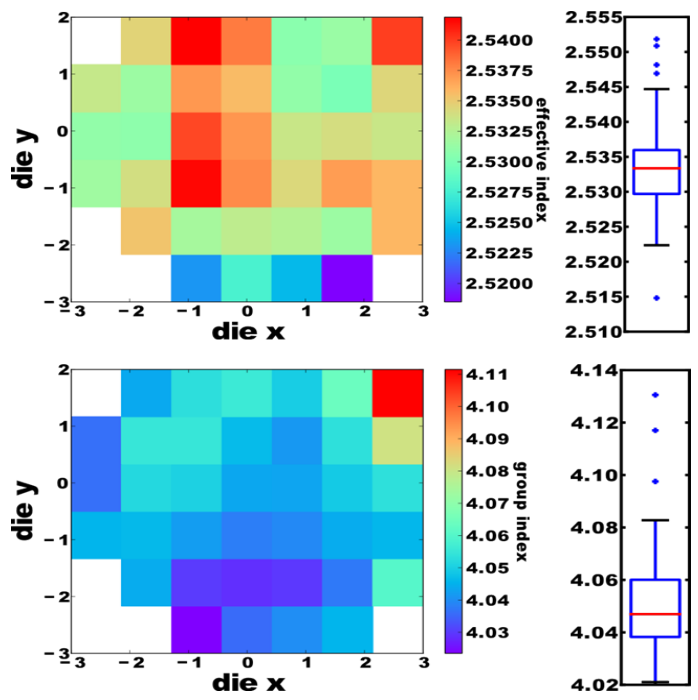

Fig. 10: Wafer map and distribution plot of $602 \mathrm{~nm}$ wide fabricated waveguide at $1550 \mathrm{~nm}$ (a) $n_{\text {eff }}$ and (b) $n_{g}$
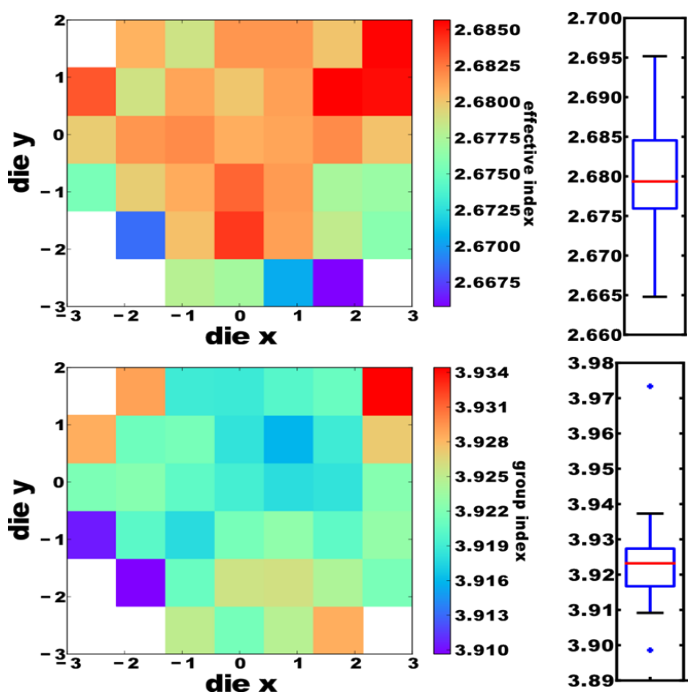

Fig. 11: Wafer map and distribution plot of $805 \mathrm{~nm}$ wide fabricated waveguide at $1550 \mathrm{~nm}$ (a) $n_{\text {eff }}$ and (b) $n_{g}$

the local variability of the effective index. It does not perturb the waveguide of local pattern density, which is essential in a high-contrast waveguide platform such as silicon photonics. This method can be applied to the TM polarization also and can be extended to other waveguide platforms as well.

\section{ACKNOWLEDGMENT}

The authors acknowledge Yanlu Li and Bendix Schneider for useful discussions, Liesbet Van Landschoot for SEM images and imec $200 \mathrm{~mm}$ p-line for device fabrication.

\section{REFERENCES}

[1] S. Selvaraja, W. Bogaerts, P. Dumon, D. Van Thourhout, and R. Baets, "Subnanometer linewidth uniformity in silicon nanophotonic waveguide devices using cmos fabrication technology," Selected Topics in Quantum Electronics, IEEE Journal of, vol. 16, no. 1, pp. 316-324, Jan 2010.

[2] W. Bogaerts, S. Selvaraja, P. Dumon, J. Brouckaert, K. De Vos, D. Van Thourhout, and R. Baets, "Silicon-on-insulator spectral filters fabricated with cmos technology," Selected Topics in Quantum Electronics, IEEE Journal of, vol. 16, no. 1, pp. 33-44, Jan 2010.

[3] W. Bogaerts, S. Pathak, A. Ruocco, and S. Dwivedi, "Silicon photonics non-resonant wavelength filters: comparison between awgs, echelle gratings, and cascaded mach-zehnder filters," pp. $93650 \mathrm{H}-93650 \mathrm{H}-12$, 2015. [Online]. Available: http://dx.doi.org/10.1117/12.2082785

[4] S. Dwivedi, H. D'heer, and W. Bogaerts, "Maximizing fabrication and thermal tolerances of all-silicon fir wavelength filters," Photonics Technology Letters, IEEE, vol. 27, no. 8, pp. 871-874, April 2015.

[5] J. Jágerská, N. L. Thomas, R. Houdré, J. Bolten, C. Moormann, T. Wahlbrink, J. Ctyroký, M. Waldow, and M. Först, "Dispersion properties of silicon nanophotonic waveguides investigated with fourier optics," Opt. Lett., vol. 32, no. 18, pp. 2723-2725, Sep 2007. [Online]. Available: http://ol.osa.org/abstract.cfm?URI=ol-32-18-2723

[6] E. Dulkeith, F. Xia, L. Schares, W. M. J. Green, and Y. A. Vlasov, "Group index and group velocity dispersion in silicon-on-insulator photonic wires," Opt. Express, vol. 14, no. 9, pp. 3853-3863, May 2006.

[7] X. Chen, Z. Li, M. Mohamed, L. Shang, and A. R. Mickelson, "Parameter extraction from fabricated silicon photonic devices," Appl. Opt., vol. 53, no. 7, pp. 1396-1405, Mar 2014. [Online]. Available: http://ao.osa.org/abstract.cfm?URI=ao-53-7-1396

[8] A. Arbabi and L. L. Goddard, "Measurements of the refractive indices and thermo-optic coefficients of si3n4 and siox using microring resonances," Opt. Lett., vol. 38, no. 19, pp. 3878-3881, Oct 2013. [Online]. Available: http://ol.osa.org/abstract.cfm?URI=ol-38-19-3878

[9] W. Bogaerts, P. De Heyn, T. Van Vaerenbergh, K. De Vos, S. Kumar Selvaraja, T. Claes, P. Dumon, P. Bienstman, D. Van Thourhout, and R. Baets, "Silicon microring resonators," Laser \& Photonics Reviews, vol. 6, no. 1, pp. 47-73, 2012. [Online]. Available: http://dx.doi.org/10.1002/lpor.201100017

[10] S. Dwivedi, T. V. Vaerenbergh, A. Ruocco, T. Spuesens, P. Bienstman, P. Dumon, and W. Bogaerts, "Measurements of effective refractive index of soi waveguides using interferometers," in Advanced Photonics 2015. Optical Society of America, 2015, p. IM2A.6.

[11] B. Tatian, "Fitting refractive-index data with the sellmeier dispersion formula," Appl. Opt., vol. 23, no. 24, pp. 4477-4485, Dec 1984. [Online]. Available: http://ao.osa.org/abstract.cfm?URI=ao-23-24-4477

[12] G. Ghosh, M. Endo, and T. Iwasaki, "Temperature-dependent sellmeier coefficients and chromatic dispersions for some optical fiber glasses," Lightwave Technology, Journal of, vol. 12, no. 8, pp. 1338-1342, Aug 1994.

[13] B. J. Frey, D. B. Leviton, and T. J. Madison, "Temperature-dependent refractive index of silicon and germanium," pp. 62 732J-62 732J-10, 2006. [Online]. Available: http://dx.doi.org/10.1117/12.672850

[14] M. Fiers, T. V. Vaerenbergh, K. Caluwaerts, D. V. Ginste, B. Schrauwen, J. Dambre, and P. Bienstman, "Time-domain and frequency-domain modeling of nonlinear optical components at the circuit-level using a node-based approach," J. Opt. Soc. Am. B, vol. 29, no. 5, pp. 896-900, May 2012.

[15] http://www.lucedaphotonics.com/.

[16] Y. Li, D. Vermeulen, Y. D. Koninck, G. Yurtsever, G. Roelkens, and R. Baets, "Compact grating couplers on silicon-on-insulator with reduced backreflection," Opt. Lett., vol. 37, no. 21, pp. 4356-4358, Nov 2012. [Online]. Available: http://ol.osa.org/abstract.cfm?URI=ol37-21-4356

[17] P. P. Absil, P. Verheyen, P. D. Heyn, M. Pantouvaki, G. Lepage, J. D. Coster, and J. V. Campenhout, "Silicon photonics integrated circuits: a manufacturing platform for high density, low power optical i/o's," Opt. Express, vol. 23, no. 7, pp. 9369-9378, Apr 2015. 